\title{
Prescribing practices in the treatment of depression among psychiatrists in Oman
}

Marwa A. Albalushi, MD, OMSBpsych, Mandhar. H Almaqbali, MD, OMSBpsych, Salim K. Al-Huseini, MD, OMSBpsych, Hamed N. Alsinawi, MD, FRCPsych.

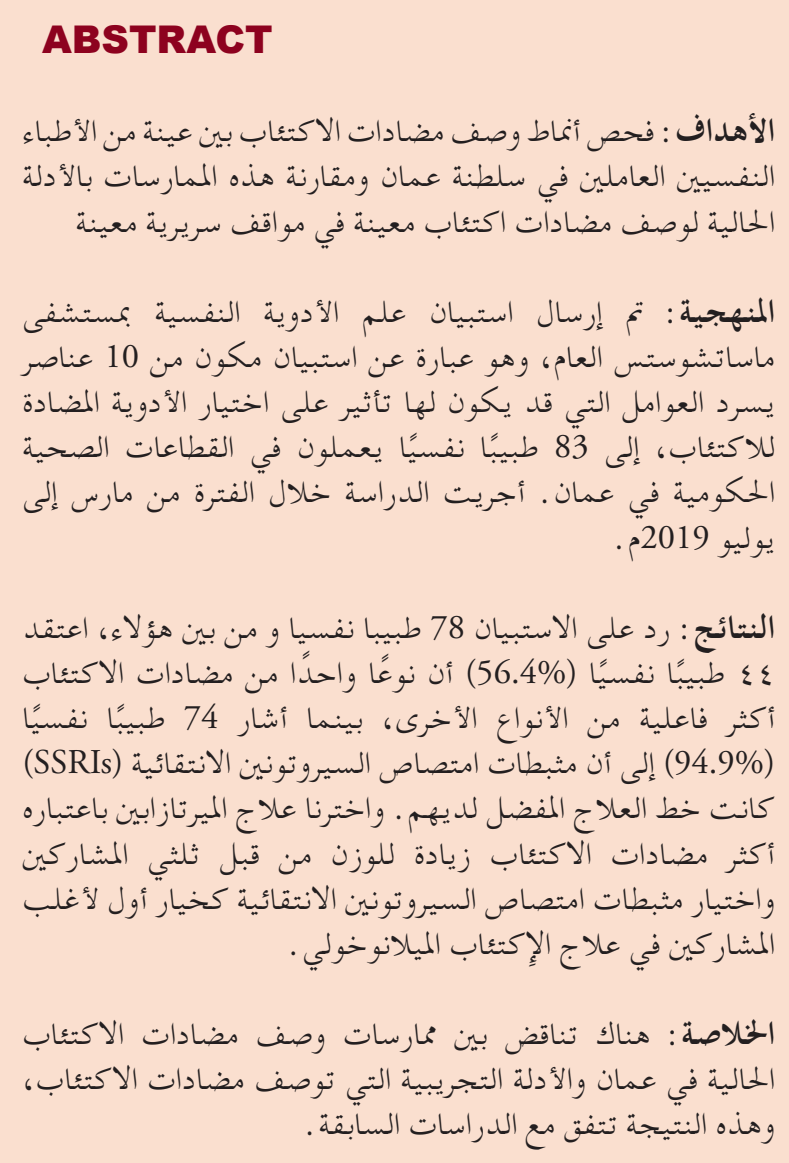

Objectives: To examine the prescribing patterns of antidepressants among a sample of psychiatrists working in Oman and to compare these practices to the current evidence for prescribing specific antidepressant in particular clinical situations.

Methods: This retrospective cross sectional study. Massachusetts General Hospital Psychopharmacology Questionnaire, a 10-item questionnaire listing factors that might have influenced the choice of antidepressant medication, was sent to 83 psychiatrists working in governmental health sectors in Oman. The study was done from March to July 2019.
Results: A total number of 78 psychiatrists responded to the questionnaire. Of these, 44 of the psychiatrists (56.4\%) believed that one type of antidepressant is more efficacious than others, while 74 psychiatrists $(94.9 \%)$ indicated that selective serotonin reuptake inhibitors (SSRIs) were their first-line treatment preference. Mirtazapine was chosen as the most likely antidepressant to cause weight gain by two-thirds of the participants. For the treatment of anxious depression and depression with melancholic feature, SSRIs were the first choice of treatment for $64.1 \%$ and $7 \%$ of respondents, respectively. For depression with atypical features, $42.3 \%$ indicated that a monoamine oxidase inhibitor would be their first option.

Conclusion: There is a discrepancy between the current antidepressant prescribing practices in Oman and empirical antidepressant-prescribing evidence, and this finding is consistent with previous studies.

Neurosciences 2021; Vol. 26 (2): 152-157 doi: 10.17712/nsj.2021.2.20200110

From the Department of Psychiatry (Al-Balushi, AlMaqbali), Sohar Hospital, Sohar, Department of Behavioral Medicine (AlSinawi), College of Medicine and Health Sciences, Sultan Qaboos University, Muscat, Ministry of Health (Al-Huseini), Oman

Received 13th August 2020. Accepted 19th October 2020.

Address correspondence and reprint request to: Dr. Mandhar almaqbali, Department of Psychiatry, Sohar Hospital, Sohar, Oman. E-mail:omanlion84@yahoo.com

ORCID ID: https://orcid.org/0000-0001-8891-9254

$\mathrm{D}$ epression is a serious and a major public health problem around the world. According to the World Health Organization (WHO), depression is the fourth leading cause of disability worldwide and it is projected that by 2030 to be the leading cause of disease burden, ${ }^{1}$ There are different modalities to treat depression including self-help, exercise, psychotherapy and counseling, however antidepressants remain the mainstay of treatment for moderate to severe 
depressive symptoms. Most antidepressants alleviate depression by affecting the neurotransmitters levels, mainly the monoamines (norepinephrine, serotonin, and dopamine). The first antidepressants used were monoamine oxidase inhibitors (MAOIs), followed by tricyclic antidepressants (TCAs), such as amitriptyline and imipramine. These two groups of drugs were widely used until the introduction of fluoxetine as the first selective serotonin reuptake inhibitors (SSRI) in $1978 .{ }^{2}$ Recently, different types of antidepressant appeared in the markets including venlafaxine and duloxetine which are work on reuptake of both serotonin and norepinephrine neurotransmitters so called (SNRIs) and the atypical antidepressant such as bupropion and mirtazapine. ${ }^{3}$

Generally, antidepressants are all equally effective in treating depression despite the difference in mechanism of action. ${ }^{3}$ The NICE guidelines recommend that SSRIs should be used as a first-line pharmacological treatment for treating depression, for no reason other than better tolerability to side effects. It is well known that TCA are associated with various side effects ranging from troublesome anticholinergic side effects to the more serious arrythmia and cardiovascular side effects. The MAOIs, are well-known for their interaction with certain foods and increase risk for hypertensive crisis. However, despite being equally effective, among the same group of SSRIs specific drugs are preferred in certain clinical situations and in particular depressive subtypes. In addition, specific antidepressant agents within the same class are associated with increased risk of certain side effects. For example, previous studies have shown that paroxetine is associated with weight gain and higher incidence of sexual dysfunction, and sertraline is more associated with diarrhea comparing to other SSRIs. ${ }^{4}$

As a general rule, and with increased numbers of antidepressant available in the markets, psychiatrists prefer to select antidepressants that are more tolerable and have a reduced risk of harm following overdose. However, there are multiple other factors that influence the decision making. These can be divided into patientspecific factors, such as patient preference, patient age, comorbid medical illness, and drug-specific factors, such as cost, tolerability, safety following overdose. ${ }^{5}$

Disclosure. Authors have no conflict of interests, and the work was not supported or funded by any drug company.
Few studies have been conducted to examine antidepressant prescribing practices. We only identified 5 studies which focused exclusively on psychiatrists: Olfson and Klerman, 1993; Olfson et al., 1998, Moore et al., 2002, Baboolal, 2002, Petersen et al., 2002. ${ }^{6-10}$ The first 2 studies did not examine prescribing practices of a specific antidepressant agents. The third study, conducted in Trinidad by Moore et $\mathrm{al}^{8}$ found that the TCAs, mainly amitriptyline, were the most prescribed antidepressants among outpatients in psychiatric clinics in Trinidad and that fluoxetine was the only SSRI prescribed in these clinics. The other 2 studies by Baboolal, 2002, and Peterson et al., 2002, were more focused on individualized drugs. The study by Baboolal surveyed 31 psychiatrists in Trinidad and Tobago using a self-administered, extensive questionnaire and Peterson et al, 2002, surveyed 450 psychiatrists in Massachusetts, USA. ${ }^{9,10}$ In both studies, psychiatrists were asked about their prescribing practices and the results were compared with empirical evidence from the scientific literature. The findings of both studies were significant and highlighted a discrepancy between the empirical evidence and clinical practices and suggested that other factors influence clinicians and their medication choices in the treatment of depression. ${ }^{10,11}$

This study aims to examine the prescribing patterns of antidepressants among a sample of psychiatrists working in Oman and to compare these practices to the current evidence for prescribing specific antidepressant in a particular situations.

Methods. This cross-sectional study was carried out from March to July 2019. The prescribing patterns of antidepressants were assessed using the Massachusetts general hospital psychopharmacology questionnaire. This is a 10 -item questionnaire that was designed by experts in psychopharmacology. The questionnaire test participants' knowledge on the following domains: 3 main domains: the effectiveness and the first line antidepressant preference in the treatment of the major depressive disorder; certain side effects and most related agents, and the preferred drug for particular depressive subtypes.

Inclusion criteria for the study encompass board certified psychiatrists and 3rd year and above psychiatry program residents working in private or governmental sectors in Oman. Only those psychiatrists who declined the invitation letter or did not respond to the e-mail of invitation to participate in the study were excluded. Therefore, all psychiatrists working--in Oman and senior psychiatry residents (Year 3 and above 
psychiatric residents at Oman medical specialty board psychiatry program) were invited to participate in the study and were asked to complete a self-administered questionnaire. Psychiatrists working in Muscat received the questionnaire by hand, whereas those working outside Muscat were contacted first by phone and the questionnaires were subsequently sent to them via email.

Ethical approval. Ethical approval was obtained from the Medical Research and Ethics Committee (MREC) at Sultan Qaboos University and the study adhered to the Declaration of Helsinki (1964).

Data management. Data analysis was done using the Statistical Package for the Social Sciences (SPSS), version 22 (IBM Corp., Armonk, NY, USA). Categorized variables were described as percentages with confidence intervals (CIs). Continuous variables were presented as means with standard deviation (SD) or medians with an inter-quartile range. To assess the relationships between the variables a univariate analysis Chi-square test, the t-test and analysis of variance (ANOVA) were used.

Results. A total of 83 questionnaires were sent out and 78 were returned completed ( $93 \%$ response rate). Of the total respondents, 34 psychiatrists $(43.6 \%)$

Table 1 - Participants' choice of preferred antidepressant agents in various clinical scenarios and depression subtypes.

\begin{tabular}{lc}
\hline Clinical scenario or depression subtype & Preferred agent (\%) \\
\hline Anxious depression & SSRIs (64.1) \\
Depression with prominent insomnia & Mirtazapine (75.0) \\
Depression with atypical features & MAOIs (42.3) \\
Depression with melancholic features & SSRIs (39.7) \\
\hline \multicolumn{2}{c}{ MAOIs - Monaoamine Oxidase Inhibitors, SSRIs - Selective } \\
\multicolumn{2}{c}{ Serotonin Reuptake Inhibitors } \\
\hline
\end{tabular}

indicated a belief that all antidepressants are equally effective, whereas 44 psychiatrists $(56.4 \%)$ believed that one type of antidepressant was more efficacious than the others. Approximately $40 \%$ of the psychiatrists in this study indicated that SSRIs were being most efficacious, while $9 \%$ indicated that tricyclic antidepressants were more efficacious than other antidepressants. Moreover, two-thirds of the participants chose SSRIs as the first-line therapy for depression.

With regards to side effects, most of psychiatrists believed that mirtazapine $(75 \%)$ and paroxetine $(17.9 \%)$ were the most likely antidepressants to cause weight gain. Moreover, paroxetine was noted by $53 \%$ of the participants as the antidepressant to be most commonly associated with sexual dysfunction and by $80 \%$ as the agent most commonly related to discontinuation syndrome. It was believed by $22 \%$ and $46 \%$ of psychiatrists that fluoxetine was related to sexual dysfunction and agitation, respectively. Notably, only $1 \%$ of participants thought that venlafaxine was associated with sexual dysfunction. Figure 1 shows the participants' responses regarding antidepressants' side effects and what they believed was the most likely associated agent.

The MAOIs and SSRIs were preferred by $42.3 \%$ and $33.3 \%$ of the psychiatrist, respectively, as the first choice in cases of depression with atypical features. Moreover, SSRIs were the first choice for $64.1 \%$ of participants in cases of anxious depression and for $39.7 \%$ in cases with depression with melancholic features. Two-thirds of psychiatrists preferred to prescribe mirtazapine for depression associated with prominent insomnia. Table 1 summarizes the participants' choices of antidepressants in various clinical situations and depression subtypes.

Discussion. This study aimed to provide information regarding the current antidepressant

Table 2 - Summary of Peterson's study results versus the current empirical evidence.

\begin{tabular}{lcc}
\hline & $\begin{array}{c}\text { Peterson's study results (percentage of participants' } \\
\text { preference of antidepressant treatment) }\end{array}$ & Evidence (APA Guidelines 2000, revised) \\
\hline Efficacy & SSRIs (48\%) & No difference \\
Sexual dysfunction & Fluoxetine $(56 \%)$ & More common with paroxetine \\
Agitation & Fluoxetine $(52 \%)$ & Comparable to other SSRIs \\
Discontinuation syndrome & Paroxetine $(47 \%)$ & Occurs among short half-life antidepressants \\
Atypical depression & SSRIs $(57 \%)$ & MAOIs \\
Melancholic depression & SSRIs $(57 \%)$ & No specific recommendation, (pharmacotherapy \\
\multicolumn{2}{c}{ and ECT leads to good responses) }
\end{tabular}




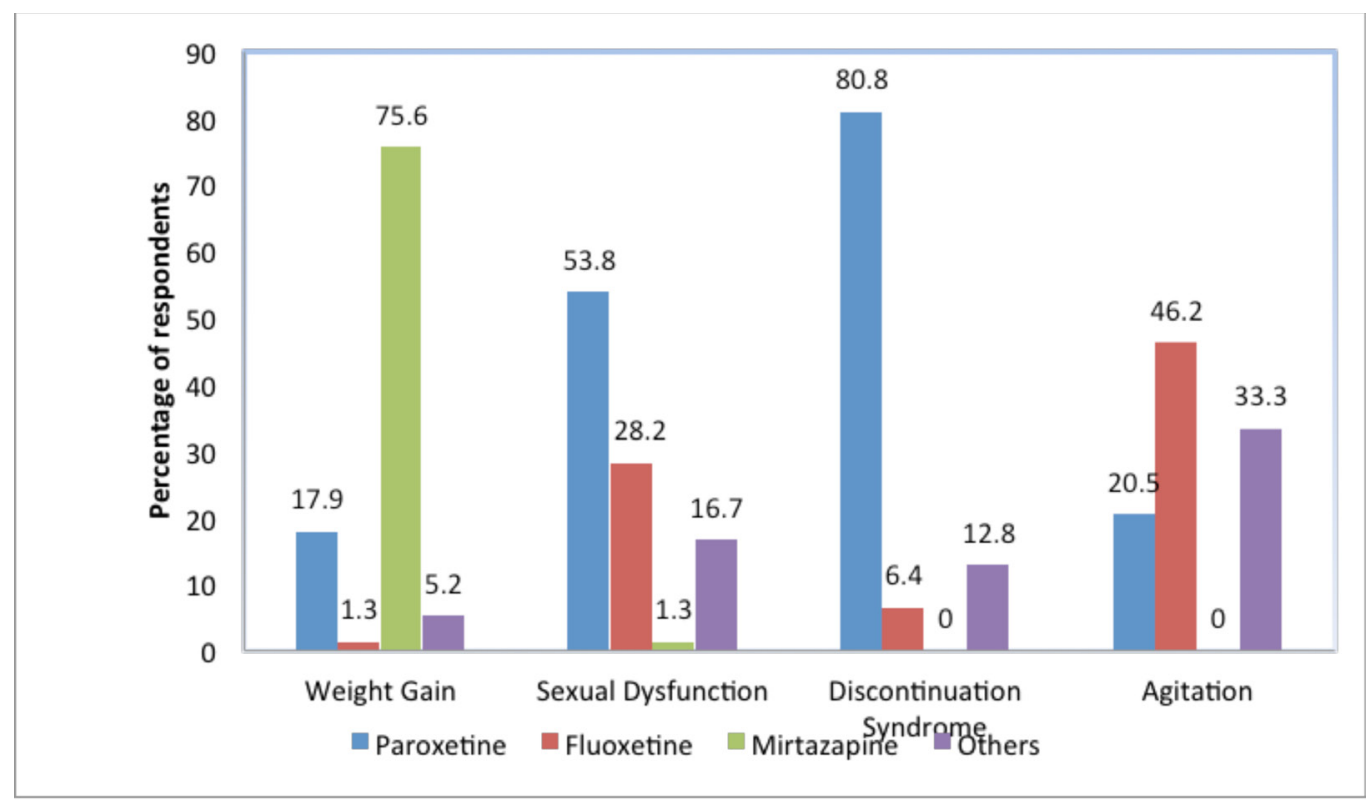

Figure 1- Participants' responses regarding the side effects of antidepressant and the most likely associated agent. *Others include antidepressants listed in the Massachusetts General Hospital Psychopharmacology questionnaire but were not mentioned in the table.

prescribing practice of psychiatrists working in Oman and will aid in comparing these practices with the available evidence globally.

This study showed that more than half of the psychiatrists in Oman believe that one type of antidepressant is more efficacious than others, with most choosing SSRIs. However, there is no evidence to support that one antidepressant is superior over another. Participants' choice of SSRI as first line treatment can possibly be explained by the fact that SSRIs are the first-line antidepressant treatment for depression. First-line treatment, however, is based on the side effect profile and tolerability of a drug rather than its efficacy. ${ }^{11}$ The Maudsley Prescribing Guidelines in Psychiatry (2018) indicates that although a network meta-analysis might suggest that some antidepressants may be more effective overall than others, this has not been consistently demonstrated in the head-to-head studies and should, therefore, be treated with caution. ${ }^{4}$ The American Psychiatric Association (APA) guideline states that: "Although some studies have suggested the superiority of one mechanism of action over another, there are no applications or robust findings to establish a clinically meaningful difference. For most patients, the effectiveness of the antidepressant medications is generally comparable between classes and within classes of medications". ${ }^{11}$ The guideline recommends that the selection of antidepressant be based on other factors such as side effect profile, safety, tolerability, patient preference, cost, history of prior response, and the presence of comorbid psychiatric and/or general medical conditions. ${ }^{11}$

In this study, paroxetine was endorsed as having the highest risk for discontinuation syndrome. According to the Maudsley Prescribing guidelines, discontinuation syndrome is seen with all antidepressants, with the possible exception of agomelatine and vortioxetine. ${ }^{4} \mathrm{It}$ should be anticipated in any patient taken antidepressant for 4-week duration or longer particularly after abrupt discontinuation of the treatment. It is more likely to occur with higher dose and short half-life drugs such as paroxetine and venlafaxine. The risk is also greater in children and adolescents, those who experience anxiety symptoms immediately after starting antidepressant therapy and those on other medications such as central-acting agents. Among the TCAs, amitriptyline and imipramine are the most common drugs to cause discontinuation syndrome, whereas all MAOIs with no exceptions can cause discontinuation syndrome. ${ }^{4}$

More than half of the responders in this study also indicated paroxetine was most commonly associated with sexual dysfunction. The APA guidelines stated that SSRI are more likely to be associated with sexual dysfunction, however erectile dysfunction, loss of libido and anorgasmia in both sexes can occur with other classes of antidepressant too. ${ }^{11}$ The Maudsley Prescribing Guidelines, based on evidence, also indicate the relatively high rates of sexual dysfunction across all 
of the SSRIs. The guidelines specified clomipramine, amitriptyline and imipramine as commonly causing sexual dysfunction among the TCAs. Similarly, venlafaxine, duloxetine, and MAOIs can cause sexual dysfunction in comparable rates to the SSRIs. ${ }^{4}$

However, there is existing evidence that support the fact that within the class of SSRIs, erectile dysfunction, vaginal lubrication difficulties, and decreased libido in both sexes are most common with paroxetine, particularly in the first month of therapy. ${ }^{12,13}$ MontejoGonzalex et al. also have found that paroxetine treatment was associated with higher rates of sexual side effects than other SSRIs. ${ }^{14}$ Conversely, Feiger et al ${ }^{15}$, Croft et $\mathrm{al}^{16}$ and Kavoussi et $\mathrm{al}^{17}$ have shown significantly lower rates of sexual side effects with nefazodone or Bupropion when compared to SSRIs other than fluoxetine in double-blind studies. These findings are all consistent with those reported in this study.

With regards to agitation, $46.2 \%$ of the responders in this study indicated that fluoxetine was the most common drug associated with this side-effect. This finding is not consistent with the existing literature, where it has been noted that the emergence of agitation following SSRI treatment occurs at an equal rate across all the SSRIs. ${ }^{10}$

When asked which antidepressants were associated with weight gain, most responders in this study (75.6\%) selected mirtazapine, which is partly consistent with empirical evidence. From literature review tricyclic antidepressants (TCAs) and monoamine oxidase inhibitors (MAOIs) are more likely to cause weight gain than the selective serotonin reuptake inhibitors (SSRIs) or the newer antidepressants, with the exception of mirtazapine. When it comes to weight gain, mirtazapine is considered in place between the SSRIs and the TCAs. ${ }^{18}$ The Maudsley Prescribing Guidelines conclude that mirtazapine is more highly associated with weight gain as compared to other antidepressants, with the exception of TCAs and MAOIs. ${ }^{4}$

Most psychiatrists in this study indicated that MAOIs $(42.3 \%)$ and SSRIs (33.3\%) were the best treatment for atypical depression. The evidence suggest that atypical depression is more responsive to MAOIs than TCAs. ${ }^{19}$ This is also consistent with the APA guidelines, which indicate that MAOIs have greater efficacy than TCAs in treating atypical depression. The guidelines also reported that there is some data to support the use of SSRIs, bupropion, and cognitive behavioral therapy. ${ }^{11}$

For the treatment of anxious depression, SSRIs have been indicated to be the preferred option. This is in partial agreement with the literature as it is a safer option as compared to MAOIs. The APA guidelines clearly state that MAOI are the best to treat anxious depression however the guidelines do not recommend its use as a first line treatment, instead safer options are recommended. ${ }^{11}$

With regards to melancholia, the responders in this study selected SSRIs as the preferred antidepressant for melancholic depression, which is not supported by the current literature. There are no consistent findings regarding the pharmacological treatment of melancholic depression. SSRIs are considered as less effective than (SNRIs), which in turn are less effective than the TCAs and MAOIs. The reason for TCA and MAOIs for being less effective is due to greater contribution of noradrenaline and dopamine. ${ }^{20}$ The APA guidelines says melancholia can be treated with pharmacotherapy and ECT. The guidelines do not specify a particular treatment as a first option for melancholic depression however it also conclude that serotonin-Norepinephrine reuptake inhibitors and TCAs may have an advantage over SSRIs. ${ }^{11}$

For the treatment of depression with prominent insomnia, the APA guidelines recommend mirtazapine and nefazodone as the most effective treatment and this is in line with the findings from this study, with $75.6 \%$ of the respondents selecting mirtazapine as the best option in such cases. An example of previous studies that compared prescribing practice of psychiatrists in treatment of depression against empirical evidence is Paterson's study in 2002 which showed presence of some degree of discrepancy between psychiatrists' prescribing pattern and the most current evidence. Peterson's study results as compared to empirical evidence are illustrated in Table 2.

Nevertheless, in this study, most of the participants' responses were consistent with the current empirical evidence, however, there were also some discrepancies. These might be attributed to several factors, especially those related to the specific medication and the particular case. We as a healthcare professional are recommended to base our practices on scientific knowledge, however, clinical decisions in patient care are often based on more than just the results of controlled experiments. ${ }^{21}$ Rush and Prien addressed factors that contributed significantly to treatment decisions. According to the authors, psychiatrist must go beyond accepted scientific knowledge and other factors need to be taken into consideration. These factors are: patient context, clinical experiences and situational context, as well as the influence of antidepressant marketing and media information. $^{22}$

Other important factors are the availability of particular antidepressant drugs in specific psychiatric 
settings. For example, in Oman, the number of antidepressants available in peripheral governmental outpatient clinics throughout the country is limited. This, in turn, has a big influence on the choice of antidepressant, as psychiatrists may have limited options as compared to the wide range of antidepressants available in the capital city, namely Sultan Qaboos University Hospital, Almasarra Hospital and the Armed Forces hospital.

In this study, the characteristics of the respondents (age, year of graduation, duration of practice, etc.) or the type of practice (outpatient, inpatient, academic setting, etc.) were not the focus of the study, therefore, future research might be needed to explore these factors, and to screen for clinical and situational factors of prescribing antidepressants as well. The growing private healthcare sector in Oman is another area of practicing psychiatry which should be considered for future research.

Limitation. The sample size is relatively small owing to low number of psychiatrists working in Oman as mental health is developing in the country. Involvement of psychiatrists working in a similar stetting such as those working in the gulf countries can overcome this limitation. Moreover, some antidepressants was not represented in the questionnaire. Designing a new tool that gives more choices for psychiatrists in various clinical situations, may reflect more accurate prescribing practices of antidepressants than the current tool.

In conclusion, as found in this study, a high level of congruence between the survey responses and current evidence indicated a good awareness and knowledge level among the participants. In order to avoid discrepancies between prescribing practices and current literature on antidepressants, establishing local prescribing guidelines that can be easily accessed by all psychiatrists in different hospitals throughout Oman is recommended. In addition, psychopharmacology courses to train psychiatrists on the recent prescribing guidelines imperative.

\section{References}

1. Lépine JP, Briley M. The increasing burden of depression. Neuropsychiatr Dis Treat 2011; 7: 3-7.

2. López-Muñoz F, Alamo C. Monoaminergic neurotransmission: the history of the discovery of antidepressants from 1950s until today. Curr Pharm Des 2009; 15: 1563-1586.

3. Depression: FDA-approved medications may help. U.S. Food and Drug Administration. From: www.fda.gov/ForConsumers/ ConsumerUpdates/ucm095980.htm. Accessed: 12 April 2020.

4. Taylor DM, Barnes TRE, Young AH. The Maudsley Prescribing Guidelines in Psychiatry, 13th Edition. John Wiley \& Sons; 2018 May 14: 258-344.
5. Zimmerman M, Posternak M, Friedman M, Attiullah $\mathrm{N}$, Baymiller S, Boland R, et al. Which factors influence psychiatrists' selection of antidepressants? Am J Psychiatry 2004; 161: 1285-1289.

6. Olfson M, Klerman GL. Trends in the prescription of antidepressants by office-based psychiatrists. Am J Psychiatry 1993; 150: 571-577.

7. Olfson M, Marcus SC, Pincus HA, Zito JM, Thompson JW, Zarin DA. Antidepressant prescribing practices of outpatient psychiatrists. Arch Gen Psychiatry 1998; 55: 310-316.

8. Moore S, Jaime LK, Maharajh H, Ramtahal I, Reid S, Ramsewak FS, et al. The prescribing of psychotropic drugs in mental health services in Trinidad. Rev Panam Salud Publica 2002; 12: 207-214.

9. Baboolal N. Prescribing practices in the treatment of depression: A survey among psychiatrists and other doctors providing psychiatric care in Trinidad and Tobago. The Internet Journal of Third World Medicine 2002; 1: 1-10.

10. Petersen T, Dording C, Neault NB, Kornbluh R, Alpert JE, Nierenberg AA, et al. A survey of prescribing practices in the treatment of depression. Prog Neuropsychopharmacol Biol Psychiatry 2002; 26: 177-187.

11. American Psychiatric Association. Practice Guideline for the Treatment of Patients With Major Depressive Disorder. From:https://psychiatryonline.org/pb/assets/raw/sitewide/ practice_guidelines/guidelines/mdd.pdf. Accessed: 12 April 2020

12. Rosen RC, Lane RM, Menza M. Effects of SSRIs on sexual function: a critical review. J Clin Psychopharmacol 1999; 19: 67-85.

13. Goldstein BJ, Goodnick PJ. Selective serotonin reuptake inhibitors in the treatment of affective disorders-III. Tolerability, safety and pharmacoeconomics.JPsychopharmacol 1998; 12: S55-S87.

14. Montejo-González AL, Llorca G, Izquierdo JA, Ledesma A, Bousońo M, Calcedo A, et al. SSRI-induced sexual dysfunction: fluoxetine, paroxetine, sertraline, and fluvoxamine in a prospective, multicenter, and descriptive clinical study of 344 patients. J Sex Marital Ther 1997; 23: 176-194.

15. Feiger A, Kiev A, Shrivastava RK, Wisselink PG, Wilcox CS. Nefazodone versus sertraline in outpatients with major depression: focus on efficacy, tolerability, and effects on sexual function and satisfaction. J Clin Psychiatry 1996; 57 Suppl 2: 53-62.

16. Croft H, Settle E Jr, Houser T, Batey SR, Donahue RM, Ascher JA. A placebo-controlled comparison of the antidepressant efficacy and effects on sexual functioning of sustained-release bupropion and sertraline. Clin Ther 1999; 21: 643-658.

17. Kavoussi RJ, Segraves RT, Hughes AR, Ascher JA, Johnston JA. Double-blind comparison of bupropion sustained release and sertraline in depressed outpatients. J Clin Psychiatry 1997; 58 : 532-537.

18. Fava M. Weight gain and antidepressants. J Clin Psychiatry 2000; 61: 37-41.

19. Pae CU, Tharwani H, Marks DM, Masand PS, Patkar AA. Atypical depression: a comprehensive review. CNS Drugs 2009; 23: 1023-1037.

20. Psychiatric times. (2017) An update on melancholia [online] [Accessed on 20th April2020]. Retrieved from: https://www. psychiatrictimes.com/cme/update-melancholia/page/0/4

21. Malterud $K$. The art and science of clinical knowledge: evidence beyond measures and numbers. Lancet 2001; 358: 397-400.

22. Rush AJ, Prien RF. From scientific knowledge to the clinical practice of psychopharmacology: can the gap be bridged? Psychopharmacol Bull 1995; 31: 7-20. 